\title{
Involvement of type- $f$ thioredoxins during germination and early seedling development and in response to oxidative stress in Arabidopsis thaliana
}

\begin{tabular}{|c|c|}
\hline Journal: & Botany \\
\hline Manuscript ID & cjb-2017-0223.R2 \\
\hline Manuscript Type: & Article \\
\hline Date Submitted by the Author: & 19-Apr-2018 \\
\hline Complete List of Authors: & $\begin{array}{l}\text { Djoumad, Abdelmadjid; Universite de Sherbrooke Faculte des Sciences, } \\
\text { Biologie } \\
\text { Villette, Solange; Universite de Sherbrooke Faculte des Sciences, Biologie } \\
\text { Isayenka, Iauhenia; Universite de Sherbrooke Faculte des Sciences, } \\
\text { Biologie } \\
\text { Beaudoin, Nathalie; Universite de Sherbrooke Faculte des Sciences, } \\
\text { Biologie }\end{array}$ \\
\hline $\begin{array}{r}\text { Is the invited manuscript for } \\
\text { consideration in a Special } \\
\text { Issue? : }\end{array}$ & N/A \\
\hline Keyword: & $\begin{array}{l}<\mathrm{i}>\text { Arabidopsis thaliana }</ \mathrm{i}>\text {, germination, oxidative stress, type- }<\mathrm{i}>\mathrm{f}</ \mathrm{i}> \\
\text { thioredoxin, UV-C }\end{array}$ \\
\hline
\end{tabular}

\section{SCHOLARONE ${ }^{m}$ \\ Manuscripts}


TITLE: Involvement of type- $f$ thioredoxins during germination and early seedling development and in response to oxidative stress in Arabidopsis thaliana

AUTHORS: Abdelmadjid Djoumad ${ }^{1}$, Solange Villette ${ }^{1}$, lauhenia Isayenka, Nathalie Beaudoin*.

${ }^{1}$ These authors contributed equally to this paper and are listed alphabetically.

* Corresponding author.

\section{AUTHORS AFFILIATIONS:}

Abdelmadjid Djoumad, Département de Biologie, Université de Sherbrooke, 2500 Boul. de l'université, Sherbrooke, QC, J1K 2R1 Canada; seyes73@yahoo.fr

Solange Villette, Département de Biologie, Université de Sherbrooke, 2500 Boul. de l'université, Sherbrooke, QC, J1K 2R1 Canada; solange.villette@neuf.fr

lauhenia Isayenka, Département de Biologie, Université de Sherbrooke, 2500 Boul. de l'université, Sherbrooke, QC, J1K 2R1 Canada; iauhenia.isayenka@usherbrooke.ca

\section{*Corresponding author:}

Nathalie Beaudoin, Département de Biologie, Université de Sherbrooke, 2500 Boul. de l'université, Sherbrooke, QC, J1K 2R1 Canada; Tel : 1-819-821-8000; Fax :1- 819-821-8049;

nathalie.beaudoin@usherbrooke.ca 


\begin{abstract}
Thioredoxins (TRXs) are important components of redox-regulated systems involved in maintaining and regulating the cellular redox environment during growth and development and in response to abiotic and biotic stress. Plants possess several types of TRX localized in various compartments, including five different types of chloroplastic TRXs. Arabidopsis thaliana contains two chloroplastic type- $f$ TRXs, TRX $f 1$ and TRX f2, involved in the regulation of light-dependent activation of photosynthesis and carbon metabolism. However, little is known about their role during development and in response to stress. We used single and double mutants of $T R X f 1$ and $T R X f 2$ to assess their roles during seed germination and seedling development. Loss of both TRX $f 1$ and TRX $f 2$ induced faster germination and seedling development associated with reduced sensitivity to ABA. This suggests that type- $f$ TRXs negatively regulate seed germination and seedling development. Moreover, double mutant seedlings were more sensitive to methyl viologen and UV-C irradiation than WT seedlings, indicating that loss of type- $f$ TRXs increases sensitivity to oxidative stress. Overall, these results indicate that type- $f$ TRXs contribute to maintaining an adequate cellular environment required for a tight regulation of germination and a better tolerance to oxidative stress.
\end{abstract}

Keywords: Arabidopsis thaliana; germination; oxidative stress; type- $f$ thioredoxin; UV-C 


\section{Résumé}

Les thiorédoxines (TRX) sont des enzymes d'oxydoréduction qui régulent l'état redox cellulaire pendant le développement et en réponse au stress. Les plantes possèdent plusieurs types de TRX dans différents organites cellulaires, incluant cinq TRX chloroplastiques. Arabidopsis thaliana possède deux TRX chloroplastiques de type $f$ impliquées dans la photosynthèse et le métabolisme carboné. Cependant, les rôles des $\operatorname{TRX} f$ pendant le développement et en réponse au stress sont peu connus. Pour préciser ces rôles, de simples et double mutants de $T R X f 1$ et $T R X f 2$ ont été analysés pendant la germination et le développement des plantules. La perte des deux isoformes a accéléré la germination et le développement des plantules tout en diminuant la sensibilité à l'ABA, suggérant que les TRX $f$ régulent négativement la germination et le développement des plantules. De plus, les plantules des doubles mutants étaient davantage sensibles au méthyle viologène et aux rayons UV-C que les plantules sauvages, démontrant que l'absence des TRX $f$ augmente la sensibilité au stress oxydatif. Ces résultats suggèrent que les TRX de type $f$ contribuent à maintenir un environnement cellulaire qui favorise un contrôle rigoureux de la germination et une plus grande tolérance au stress oxydatif.

Mots clés: Arabidopsis thaliana; germination; stress oxydatif; thiorédoxine de type-f; UV-C 


\section{Introduction}

Plant cells continuously produce reactive oxygen species (ROS), such as superoxides and peroxides, during the processes of photosynthesis and respiration. While precise levels of ROS are important in regulating many plant growth- , stress- and defence-related pathways (Ray et al. 2012; Das et al. 2015), high concentrations and continued exposure to ROS perturb the cellular redox balance. This oxidative environment can alter important signaling pathways and cause damages to cellular components that lead to oxidative stress (Ray et al. 2012; Das et al. 2015). Perturbations in the redox balance can have major impacts on cell functions and may ultimately lead to senescence and programmed cell death (PCD) events (Das et al. 2015; Petrov et al. 2015). Therefore, a tight regulation of the cellular redox state is essential to be able to protect cells from oxidative damage and transduce ROS signals into proper cell responses. In addition to antioxidant mechanisms that protect cells from excessive ROS production, cells produce redox-sensitive proteins that are modified in response to the cellular redox state to activate or inactivate redox-sensitive pathways. Redox active proteins have been implicated in most developmental, physiological and cellular processes, including cell proliferation, seed development and germination, response to light, photosynthesis and carbon metabolism, synthesis of starch, primary and secondary metabolism, and response to abiotic and biotic stress (Das et al. 2015; Petrov et al. 2015; Rouhier et al. 2015). These redox modifications are often directly or indirectly mediated by ROS using reversible reducing systems such as thioredoxins (Montrichard et al. 2009; Das et al. 2015; Rouhier et al. 2015).

Thioredoxins (TRXs) are small 12 to $14-\mathrm{kDa}$ disulphide reductase proteins involved in maintaining and regulating the cellular redox environment through the reversible reduction of disulfide bonds (Montrichard et al. 2009; Buchanan et al. 2012). Mammalian cells contain only two TRXs that are both essential for life (Matsui et al. 1996; Nonn et al. 2003). They have been implicated in several diseases and were shown to protect cells against apoptosis. Their redox regulatory roles are crucial in several redoxregulated signaling cascades and are essential for cell function, cell proliferation and antioxidant defense (Nordberg and Arnér 2001; Lu and Holmgren 2014). In contrast to their animal counterparts, plant TRXs exist in several types $(f, m, x, y, z, o, h)$ that are localized in various cellular compartments, including 
cytosol, mitochondria, nucleus and plastids (Bohrer et al. 2012; Buchanan et al. 2012; Meyer et al. 2012; Balsera et al. 2014; Belin et al. 2015). Type- $f$ and $-m$ TRXs were the first of the five types of plastidic TRXs to be characterized (Rouhier et al. 2015). They have been shown to act as messengers in the Fdx (ferredoxin)/TRX system by relaying the redox signal from Fdx:TRX reductase to target enzymes (Dai et al. 2007). Both types are involved in the regulation of light-dependent activity of enzymes involved in photosynthesis and carbon metabolism (Michelet et al. 2013; Rouhier et al. 2015). For example, experimental evidence from mutant analyses suggests that type- $f$ and $-m$ TRXs can both activate fructose1,6-phosphatase (FBPase) in vivo, indicating some overlap in substrate specificity between these isoforms (Cheng et al. 2014; Okegawa and Motohashi 2015). More specifically, type-m TRX participates in the inactivation of glucose-6-phosphate dehydrogenase, the first enzyme in the oxidative pentose phosphate cycle (Cheng et al. 2014). Type-f TRXs can regulate several chloroplastic enzymes that participate in the Calvin-Benson cycle, ATP synthesis and fatty acid synthesis (Michelet et al. 2013; Cheng et al. 2014; Rouhier et al. 2015). While most single TRX $f$ or TRX $m$ mutants display little or no visible phenotypic changes under standard growth conditions (as described below), reduced or loss of expression of both TRX $f$ and TRX $m$ leads to a detectable phenotype mainly linked to carbohydrate content (Thormählen et al. 2013; Yoshida et al. 2015; Naranjo et al. 2016).

Type- $f$ TRX alone can activate ADP-glucose pyrophosphorylase (AGPase), a key enzyme in starch synthesis (Thormählen et al. 2013). However, while transplastomic overexpression of TRX $f$ in tobacco leaves stimulated starch production and increased biomass, AGPase post-translational redox activation was not increased in these plants compared to wild-type (WT) plants (Sanz-Barrio et al. 2013). How TRX $f$ overexpression can increase starch content in tobacco plants has not been elucidated so far, but TRX $f$ is the most efficient plant reductant in the regulation of several other enzymes involved in starch synthesis and degradation. These include $\alpha$-glucan water dikinase (GWD; Mikkelsen et al. 2005), dual-specificity phosphatase starch excess 4 (SEX4; Silver et al. 2013), starch synthase 1 (SS1; Skryhan et al. 2015), $\beta$ amylase 1 (BAM1; Sparla et al. 2006; Valerio et al. 2011) and $\alpha$-amylase 3 (AMY3; Seung et al. 2013). 
In addition to their important role in carbon metabolism, type- $f$ TRXs would also be involved in controlling ROS metabolism and the cellular redox balance. Partial silencing of TRX $f$ alone in tomato was sufficient to increase levels of MDA, an indicator of ROS-induced lipid peroxidation. This suggested that partial loss of TRX $f$ increased oxidative stress, which was attributed at least in part to a decreased activity of antioxidant enzymes (Cheng et al. 2014). Similarly, more ROS $\left(\mathrm{O}_{2}{ }^{-}\right.$and $\left.\mathrm{H}_{2} \mathrm{O}_{2}\right)$ production was detected in pea leaves that were simultaneously silenced for both type- $f$ and $-m$ TRXs (Luo et al. 2012).

While most plant species studied to date contain only one isoform of type- $f$ TRX, Arabidopsis thaliana expresses two type- $f$ TRXs, TRX $f 1$ and TRX $f 2$. Arabidopsis leaves accumulate about three times more TRX $f 1$ protein than TRX f2 (Okegawa and Motohashi 2015). The general growth phenotype of Arabidopsis knockout mutants for either $T R X f 1$ or $T R X f 2$ gene has been reported to be comparable to wild-type (WT) plants, possibly because the loss of one family member can be compensated by the other member or by other types of plastidic TRXs (Thormählen et al. 2013; Yoshida et al. 2015; Naranjo et al. 2016). Similarly, double trxf1;trxf2 mutants grown in normal 16-h photoperiod were not apparently different than WT (Yoshida et al. 2015; Naranjo et al. 2016). However, the loss of TRXf1 expression alone in Arabidopsis perturbed diurnal reductive activation of AGPase, leading to a reduction in starch accumulation (Thormählen et al. 2013), an effect that was amplified in the double trxf1;trxf2 mutant (Naranjo et al. 2016). Moreover, when single trxf1 or trxf2 mutants or the double mutant were grown in short day conditions, plant growth was delayed, especially in the double mutant, and this could not be rescued by high light intensity. This phenotype was associated with lower efficiency of photosynthetic parameters and diminished reduction of Calvin-Benson enzymes (Naranjo et al. 2016).

These results show clear evidence for the involvement of Arabidopsis type- $f$ TRXs in the redox regulation of photosynthetic and carbon fixation processes. However, there is currently little information on other roles that Arabidopsis TRX $f 1$ and $f 2$ may play in the control of cellular homeostasis during developmental processes and in response to stress. In this work, we have used a genetic approach with single trxf1 and trxf2 single and double insertional mutants to investigate the implication of the Arabidopsis type- $f$ TRXs 
during germination and in response to oxidative stress. Our results show the importance of a tight balance of these redox proteins to properly regulate seed germination and response to oxidative stress.

\section{Materials and methods}

Plant material and growth conditions

For in vitro analyses, seeds from Arabidopsis thaliana wild-type (Col-0) and mutants were surfacesterilized and sown in Petri dishes containing germination medium (GM: 0.5 X Murashige and Skoog (MS) medium (Sigma-Aldrich, Oakville, ON, Canada), 1\% sucrose and 0.8\% (w/v) Difco-agar, pH 5.7). For analysis of seed germination and seedling development in response to ABA or methyl viologen (MV), 0 to $5 \mu \mathrm{M} \mathrm{ABA}$ or 0 to $1 \mu \mathrm{M} \mathrm{MV}$ was included in GM. After cold stratification at $4^{\circ} \mathrm{C}$ for 3 to 4 days, plates were transferred to a Sanyo environmental growth Chamber MLR-350 at $22^{\circ} \mathrm{C}$ under long day $(16 \mathrm{~h}$ light $/ 8 \mathrm{~h}$ darkness) photoperiod or continuous light as indicated. For plants grown in soil, seeds were either directly sown in soil or 2-week-old in vitro-grown seedlings were transferred in pots containing a mixture of soil (Agromix, Fafard, Saint-Bonaventure, QC, Canada) and vermiculite (3:1, v/v) irrigated with water and fertilized once a week with a solution $\left(1 \mathrm{~g} \mathrm{~L}^{-1}\right)$ of commercial fertilizer (20-20-20; Plant-Prod, Brampton, ON, Canada). Plants were grown in a growth chamber (Conviron MTR30, Winnipeg, MB, Canada) under long day (16 h light / $8 \mathrm{~h}$ darkness) photoperiod at an intensity of approximately $100 \mu \mathrm{mol} \mathrm{m}^{-2} \mathrm{~s}^{-1}$ with $22^{\circ} \mathrm{C}$ during the day and $19^{\circ} \mathrm{C}$ at night.

Isolation and identification of mutants

The trxf1-4 mutant was isolated from a T-DNA mutant collection transformed with a promoter-less luciferase gene graciously obtained from Dr. László Szabados (Alvarado et al. 2004). Briefly, 100 to 300 seeds from T2 seeds pools were germinated on GM for 10 to 12 days. Two mM D-Luciferin (Gold Biotechnology, St-Louis, MO, USA) was sprayed on seedlings. Luciferase activity was visualized in a custom made dark cabinet using a CCD camera (MicroMAX System, Roper Scientific, USA) and analysed with the WinView/32 software. Seedlings were then exposed to UV-C $\left(5 \mathrm{~kJ} \mathrm{~m}^{-2}\right)$ and returned to the growth chamber for $24 \mathrm{~h}$. Luciferase activity was analyzed a second time as described. Seedlings that had high 
luciferase activity before UV-C treatment but very low luciferase activity or none after UV-C irradiation were selected and transferred to soil for seed production. The location of the T-DNA insertion was performed using TAIL-PCR as described previously (Weigel and Glazebrook 2002; Alvarado et al. 2004). DNA gel blot analysis was performed to confirm single locus insertion of the T-DNA.

Other T-DNA mutant lines were obtained from the Arabidopsis Biological Resource Center, Ohio State University, with SALK_099762C corresponding to trxf1-2 and GK-020E05 corresponding to trxf2-1. Homozygous knock-out plants were identified by PCR using gene-specific primers and primers binding to the left-border of the T-DNA listed in Supplementary data, Table S1 ${ }^{1}$. Double mutants were obtained by manual crosses between single mutants. F2 seeds from self-crossed F1 plants were germinated and double homozygous plants were identified using gene-specific primers and primers binding to the leftborder of each T-DNA.

RNA extraction and quantification of gene expression

Total RNA was extracted from seedlings using RNeasy Plant Mini Kit (Qiagen, Toronto, ON, Canada). cDNA synthesis was performed using $2 \mu \mathrm{g}$ total RNA with AMV Reverse transcriptase (Promega, Madison, WI, USA) following manufacturer's instructions. Gene expression was analysed by RT-PCR using $1 \mu \mathrm{L}$ of cDNA and Taq DNA polymerase (NEB, Whitby, ON, Canada) with the specific primers listed in Suppl. data, Table S1.

Protein isolation and immunoblot analysis

Total protein was extracted from $50 \mathrm{mg}$ of Arabidopsis rosette leaves grown in long (16 h/8 h) days or seedlings (UV-C treatment) grown in continuous light conditions, as indicated. Plant material was homogenised in extraction buffer containing $50 \mathrm{mM} \mathrm{HEPES} / \mathrm{KOH}, \mathrm{pH} 7.4,5 \mathrm{mM} \mathrm{NaCl}, 10 \mathrm{mM} \mathrm{MgCl}, 2 \mathrm{mM}$ DTT (Toivola et al. 2013). Proteins were quantified using Bio-Rad Protein Assay (Bio-Rad Laboratories, Inc.

\footnotetext{
${ }^{1}$ Supplementary data are available with the article through the journal Web site at http://nrcresearchpress.com/doi/suppl/10.1139/cjb-2017-0223
} 
Mississauga, ON, Canada). Protein extracts were heated at $100^{\circ} \mathrm{C}$ for 2 min and fractionated in $15 \%$ polyacrylamide gel containing $6 \mathrm{M}$ urea. After separation, proteins were blotted on PVDF membrane (Amersham Hybond $0.45 \mu \mathrm{m}$ PVDF Blotting Membrane, GE Healthcare Life Sciences, Mississauga, ON, Canada). TRX $f 1$ and $f 2$ were detected using primary antibodies raised against TRX $f 1 / f 2$ (CLAS14-2808, Cedarlane, Burlington, ON, Canada) at $4^{\circ} \mathrm{C}$ overnight (Nikkanen et al. 2016). Primary antibodies on blots were detected by HRP-labeled goat anti-rabbit secondary antibodies (HS101, TransBionovo, Inc., Beijing, China). Protein band density from blots and Coomassie stained SDS-PAGE were analysed using ImageJ software (Schneider et al. 2012).

\section{UV-C treatment}

Seeds were germinated in continuous light. Three to 10-day-old seedlings were irradiated using 5 to $50 \mathrm{~kJ}$ $\mathrm{m}^{-2}$ with a Stratalinker (Agilent-Stratagene, model 2400, Mississauga, ON, Canada) and returned to continuous light. Epifluorescence microscopy detection of ROS was performed $24 \mathrm{~h}$ after UV-C treatment of 5-day-old seedlings incubated for $30 \mathrm{~min}$ in $10 \mu \mathrm{M} 2^{\prime}, 7^{\prime}$-dichlorodihydrofluorescein diacetate $\left(\mathrm{H}_{2}\right.$ DCFDA) diluted in phosphate buffer saline (PBS). Detection of live and dead cells in roots of 3-day-old seedlings irradiated with UV-C ( 5 to $30 \mathrm{~kJ} \mathrm{~m}^{-2}$ ) was performed $24 \mathrm{~h}$ after treatment. Whole seedlings were stained for $5 \mathrm{~min}$ with fluorescein diacetate (FDA; $50 \mu \mathrm{M}$ ) to detect live cells (green) and propidium iodide (PI; $2.78 \mu \mathrm{M}$ ) to detect dead cells (red) and rinsed three times with water before being analysed by epifluorescence microscopy. Epifluorescence microscopy analyses were performed using Axiolmager Z1 microscope (Carl Zeiss, Toronto, ON, Canada). Total RNA extraction and cDNA synthesis were performed as described above using untreated seedlings, seedlings harvested directly after UV-C irradiation ( $0 \mathrm{~h})$ or 24 and $48 \mathrm{~h}$ after UV-C treatment. Gene expression was analysed by RT-PCR using $1 \mu \mathrm{L}$ of cDNA and Taq DNA polymerase (NEB, Whitby, ON, Canada) with the specific primers listed in Suppl. data, Table S1.Total protein was extracted from 6-day-old WT control seedlings or $24 \mathrm{~h}$ after UV-C treatment $\left(30 \mathrm{~kJ} \mathrm{~m} \mathrm{~m}^{-2}\right.$. Chlorophyll was quantified as described below $72 \mathrm{~h}$ after UV-C irradiation of 6-day-old seedlings.

\section{Quantification of chlorophyll content}


Seedlings grown on medium containing $0.5 \mu \mathrm{M} M V$ or exposed to UV-C (5 and $15 \mathrm{~kJ} \mathrm{~m}^{-2}$ ) were harvested, weighted and chlorophyll was extracted in $80 \%$ acetone for $24 \mathrm{~h}$ at $4^{\circ} \mathrm{C}$. Quantification was performed as described (Lichtenthaler 1987).

Starch detection and quantification

Starch was visualized using Lugol's or $\mathrm{I}_{2} / \mathrm{KI}$ solution as described before (Li et al. 2007). Briefly, 10-day-old seedlings were boiled in $80 \%$ ethanol $(\mathrm{v} / \mathrm{v})$, stained with Lugol's solution for $10 \mathrm{~min}$ followed by two rinses with water. Pictures of rosettes or seedlings on a light box were immediately taken using a Coolpix S8200 camera (Nikon, Mississauga, ON, Canada). Accumulation of starch granules in root-cell amyloplasts and leaf chloroplasts was visualized using a modified protocol (Ding and Friml 2010). Seedlings grown vertically were stained 3 days after germination with a 1\% Lugol's solution for 2 min and rinsed 2 times. For root apices, samples were incubated in a solution of Visikol ${ }^{\mathrm{TM}}$ (Phytosys LLC, New Brunswick, NJ, USA). Samples were analysed using Axiolmager Z1 microscope. The amount of starch was quantified in leaf tissue samples collected at the end of the light period using the amylase/amyloglucosidase method of STA20 kit (Sigma-Aldrich, Oakville, ON, Canada) or the total starch quantification method of the K-TSTA 07/11 kit from Megazyme (Megazyme International, Wicklow, Ireland), as recommended by the manufacturer.

\section{Results}

Comparison of TRX $f 1$ and TRX f2 sequences, gene expression profiles and cellular localization

Arabidopsis TRX $f 1$ and TRX $f 2$ proteins have a high degree of homology with $78 \%$ amino acid identity (Collin et al. 2003). Both proteins contain the conserved TRX active redox site identified as the short peptide sequence WC(G/P)PC that includes two conserved cysteine residues. The most important differences are observed within the chloroplast transit peptide sequences which have $59 \%$ identity, while the C-terminus that contains the active site is highly conserved with $88 \%$ identity. Expression profiles of the Arabidopsis genes coding for TRX $f 1$ (At3g02730) and for TRX f2 (At5g16400) have previously been analysed by RT-qPCR (Bohrer et al. 2012) or were obtained from the Arabidopsis eFP Browser (Winter et 
al. 2007) as presented in previous work (Belin et al. 2015). These data are summarized in Suppl. data, Fig. S1. Both genes show a conserved pattern of expression, with the TRX $f 1$ gene being expressed at higher levels than $T R X f 2$ in most tissues, except for mature seeds and pollen. Based on sequence analysis, both TRX $f 1$ and $f 2$ are predicted to be localized to the chloroplasts. Experimental evidence using ectopic expression in protoplast showed that TRX $f 1$ fused to GFP localizes to chloroplasts (Collin et al. 2003). Only indirect evidence from proteomic analyses has suggested that TRX $f 2$ also localizes to chloroplasts (Belin et al. 2015). These results were confirmed using transient expression of TRX $f 1$ or TRX $f 2$ fused to the YFP gene into protoplasts produced from light-grown Arabidopsis cell suspensions or mesophyll tissues (Suppl. data, Fig. S2). Chlorophyll fluorescence, a marker of chloroplast, and YFP fluorescence both co-localized in transformed protoplasts with no fluorescence detected in other cellular compartments or membrane. This data confirms that TRX $f 1$ and $f 2$ are specifically targeted to the chloroplasts. Isolation of trxf1 and trxf2 single and double mutants

At least three different trxf1 mutants have been described before (Thormählen et al. 2013; Yoshida et al. 2015; Naranjo et al. 2016). Only one trxf2 mutant was isolated so far (Yoshida et al. 2015; Naranjo et al. 2016). Mutant seeds from the insertional mutant trxf1-2 (Yoshida et al. 2015) and for the mutant trxf2-1 were obtained from the Arabidopsis Biological Resource Center. Plants with homozygous T-DNA insertion were selected using the primers described (Suppl. data, Table S1). We isolated another trxf1 mutant, named hereafter trxf1-4, from a collection of insertional mutants transformed with a promoter-less luciferase gene, as described in Materials and methods (Alvarado et al. 2004). Luciferase activity in this mutant was lost after UV-C treatment, suggesting that the promoter located close to the T-DNA insertion was downregulated by UV-C irradiation. Analysis of TRX f1 gene expression in UV-C treated WT seedlings and in the isolated mutant supported this hypothesis (Suppl. data, Fig. S3). The T-DNA insertion was localized using TAIL-PCR (Weigel and Glazebrook 2002; Alvarado et al. 2004) in the 5' region of the TRXf1 gene, 43 nucleotides upstream of the initiation codon. Localization of the T-DNA insertion in each of TRX $f 1$ and $T R X f 2$ genes is presented in Fig. 1 a-b. Crosses were performed between trxf1-4 and trxf2-1 homozygous mutants to produce the trxf1;trxf2 mutant, trxf1-4 being the pollen recipient plant. Loss of 
$T R X f 1$ and/or TRXf2 gene expression in single and double mutants was confirmed by PCR (Fig. 1c). Levels of type- $f$ TRX protein in leaves of mutants were evaluated by immunoblot analyses using specific anti-TRX $f$ antibodies that recognize both TRX $f 1$ and TRX $f 2$ (Nikkanen et al. 2016). As shown in Fig. 1 d, the anti$\operatorname{TRX} f$ detected a band of approximately $13 \mathrm{kDa}$ that corresponds to TRX $f 1(13.3 \mathrm{kDa})$ and TRX $f 2(14.1$ kDa) in WT leaf tissues. No type- $f$ TRXs were detected in leaf tissues of the double trxf1;trxf2 mutant while the trxf1 single mutants had about 6 to $7 \%$ of the WT level (corresponding most probably to TRX $f 2$ ), and the trxf2 single mutant had about $61 \%$ of the WT level (corresponding most probably to TRX $f 1$ ).

\section{Faster germination in trxf1;trxf2 double mutant correlates with decreased sensitivity to $A B A$}

The phenotype and development of type-f TRX single and double mutant plants grown in 16-h photoperiod were comparable to that of WT plants. There were no visible changes in leaf, stem or root size, morphology or growth pattern, and flowering occurred at the same time as WT, corroborating previous reports which indicated no phenotypic changes in single and double mutants grown in standard conditions (Thormählen et al. 2013; Yoshida et al. 2015; Naranjo et al. 2016). However, a careful analysis of germination (radicle emergence) in trxf1 and trxf2 single and double mutants showed that seeds of trxf1-2 and trxf1;trxf2 germinated faster than WT seeds in the first 2 - 3 days after stratification (Fig. 2a). However, seeds from trxf1-4 and trxf2 single mutants did not germinate faster. The different response of the trxf1-4 and trxf1-2 is intriguing. One explanation may be that the trxf1-4 mutant is somehow leaky with weak $T R X f 1$ expression specifically during germination. However, this possibility is unlikely as we have not been able to detect expression of $T R X f 1$ in trxf1-4 or in the double mutant produced by crosses between trxf1-4 and trxf2 (Fig. 1 and 5). As for the trxf2 mutant, seed germination was delayed but cotyledon greening (Suppl. data, Fig. S4) and seedling weight (Table 1) were comparable to WT. However, all trxf2 seed batches tested for this experiment had a low germination efficiency (less than $90 \%$ at day 10; Suppl. data, Table S2), which may explain the delayed germination. In contrast, seedling development (cotyledon greening) was significantly faster only in trxf1;trxf2 compared to WT or single mutant seedlings (Suppl. data, Fig. S4), supporting a contribution of both type- $f$ TRXs in early seedling development. Overall, 
these data suggest that germination is mainly accelerated by the loss of TRX $f 1$ while the combined loss of both type-f TRXs stimulates faster seedling development and growth.

Seed germination and post-germination seedling establishment are negatively regulated by ABA (Gazzarrini and McCourt 2001). Since trxf1-2 and trxf1;trxf2 seeds germinated faster, sensitivity to ABA during germination was evaluated in the single and double mutants. As shown in Fig. 2b (left panel), germination was not significantly improved on ABA for all mutants. On the other hand, cotyledon greening was significantly faster at day 5 on $1.0 \mu \mathrm{M} \mathrm{ABA}$ for trxf1-2 and the double mutant but not for the single mutants trxf1-4 and trxf2 (Fig. 2b, right panel). Germination at day 2 was significantly reduced in the trxf2 mutant on all ABA levels when compared to WT. We cannot exclude that trxf2 is indeed more sensitive to $A B A$, but as mentioned above, germination was impaired in the trxf2 mutant, which may contribute to the slower germination phenotype. Overall, these results suggest that TRX $f 1$ is the main contributor in the decreased sensitivity to ABA observed in the double mutant.

\section{Reduced starch accumulation in trxf1:trxf2 double mutant is associated with increased seedling weight}

Prior studies have shown that starch deposition was reduced in the Arabidopsis single trxf1 mutant and in the trxf1; trxf2 double mutant (Thormählen et al. 2013; Naranjo et al. 2016) while transplastomic overexpression of TRX $f$ in tobacco leaves increased production of starch (Sanz-Barrio et al. 2013; Aranjuelo et al. 2015). To corroborate these results, visualisation of starch deposition using Lugol's staining was performed in rosette and roots of single and double mutant seedlings grown in 16-h light conditions (Fig. 3). As anticipated, starch deposition was visibly reduced in the rosette of trxf1-4 and of trxf1;trxf2 double mutant (Fig. 3a). Changes in starch deposition were more apparent in root tissues, with little Lugol's staining starch in the trxf1-4 mutant and the double mutant compared to WT (Fig. 3b-c). Quantification of starch in leaf tissues at the end of the light period showed a 25 to $35 \%$ decrease in starch content that was statistically significant in trxf1-4 and trxf1;trxf2 when compared to WT (Fig. 3d).

Previous work studying natural variation of starch accumulation in different Arabidopsis accessions showed a negative correlation between starch accumulation and biomass, suggesting that investing in 
starch accumulation may decrease growth (Sulpice et al. 2009). Following this idea, we measured the biomass of 10-day-old mutant and WT seedlings (Table 1). The biomass of double mutant seedlings was $30 \%$ superior to that of WT seedlings. Some changes in starch deposition and growth were also observed when seedlings were developed in the dark and cold $\left(4^{\circ} \mathrm{C}\right)$ on sucrose-supplemented medium for three weeks (Suppl. data, Fig. S5). In these conditions, starch accumulation in mutant cotyledons was comparable to WT while the root apices of the $\operatorname{trxf1-4}$ and trxf1;trxf2 double mutants had visibly less accumulation of starch. Moreover, the etiolated hypocotyls grew $12 \%$ longer in the double mutant than in the WT seedlings. These results suggest that the loss of type- $f$ TRXs reduced the energy invested in starch deposition and increased seedling growth.

Increased sensitivity to oxidative stress induced by methyl viologen (MV) in trxf1 and trxf2 single and double mutants

Preceding work has suggested that type- $f$ TRXs may be implicated in the response to oxidative stress. Hence, Cheng et al. (2014) have provided evidence that partial loss of TRX $f$ in tomato leaves induced oxidative stress in leaves. Likewise, partial silencing of both TRX $f$ and TRX $m$ increased ROS production in pea leaves (Luo et al. 2012). However, only overexpression of TRX $m$ increased tolerance to methyl viologen (MV) in tobacco leaves while TRX $f$ overexpessing leaves responded as WT plants (Rey et al. 2013). To determine whether Arabidopsis type- $f$ TRXs are involved in the response to oxidative stress, seeds of single and double mutants were plated on medium containing various concentrations of MV, an herbicide that inhibits photosynthesis and induces oxidative stress due to the production of superoxide free radical at PSI sites via the photoreduction of dioxygen (Kim and Lee, 2003). In WT Arabidopsis plant, MV can perturb seed germination and seedling development and impair survival. As shown in Fig. 4a, seedling development (cotyledon greening) on $0.5 \mu \mathrm{M}$ and $1.0 \mu \mathrm{M} \mathrm{MV}$ was reduced in all single and double mutants when compared to WT seedlings. Oxidative stress can also induce chlorophyll degradation in MV-treated tissues, which leads to cotyledon or leaf whitening. Since type- $f$ TRXs have been shown in vitro to participate in the activation of the subunit CHLI1 of Mg-Chelatase, an enzyme involved in chlorophyll synthesis (Ikogami et al. 2007), it is possible that cotyledon whitening was also due 
to decreased chlorophyll synthesis in the trxf mutants. We quantified total chlorophyll in $0.5 \mu \mathrm{M} \mathrm{MV-}$ treated seedlings with green cotyledons and compared their chlorophyll content with untreated seedlings. The chlorophyll content in untreated trxf mutants was similar to WT (data not shown), suggesting that chlorophyll synthesis was not affected in seedlings, at least in normal growth conditions. The relative chlorophyll loss in 10-day-old seedlings was $13 \%$ higher in the double mutant grown on MV compared to WT seedlings grown in similar conditions (Fig. 4b). Overall, these results support an increased sensitivity to oxidative stress mediated by MV due to the loss of type- $f$ TRXs in Arabidopsis.

Loss of type-f thioredoxins increases sensitivity to UV-C

The trxf1-4 mutant contains a promoter-less luciferase gene inserted in the promoter region of the TRX f1 gene. This mutant was isolated based on a reduced activity of luciferase after exposure to UV-C. This suggested that the $T R X f 1$ promoter was repressed by UV-C irradiation, leading to downregulation of TRX f1. This hypothesis was confirmed using RT-PCR (Fig. 5a, upper panel; Suppl. data, Fig. S3). Gene expression of $T R X f 1$ in WT and trxf mutants seedlings after UV-C irradiation was strongly downregulated by UV-C treatment. TRX $f 2$ gene expression was also downregulated in response to UV-C, albeit to a lesser extent. As expected, no expression of $T R X f 1$ or $T R X f 2$ was detected in the corresponding mutants and double mutant. Moreover, loss of $T R X f 1$ did not alter expression of TRX $f 2$, and loss of TRX $f 2$ was not compensated by increased expression of $\operatorname{TRX} f 1$. To determine whether UV-C irradiation altered type- $f$ TRX protein levels, total protein extracted from WT seedlings that had been irradiated or not with UV-C were subjected to immunoblot analyses using the anti-TRX $f$ antibodies. As shown in Fig. 5a (lower panel), type-f TRXs were still detected $24 \mathrm{~h}$ after UV-C irradiation at a level that corresponded to about $80 \%$ of control levels. This indicates that type- $f$ TRX proteins were still abundant $24 \mathrm{~h}$ after UV-C irradiation despite a marked decrease of $\operatorname{TRX} f$ gene expression.

UV-C irradiation induces photooxidative stress associated with chlorophyll degradation and programmed cell death (PCD) in Arabidopsis seedlings (Danon and Gallois 1998; Wituszyńska et al. 2015). In animal cells, UV-C induced the expression of TRXs which would function as antioxidants to protect cells against 
oxidative stress by reduction of ROS and through redox regulation of stress signaling (Charruyer et al. 2007; Ono et al. 2012). In contrast, downregulation of gene expression of type- $f$ TRXs in response to UV-C suggests that they are not implicated in protecting cells against UV-C and the oxidative stress it may cause. On the opposite, their presence could even be detrimental to cell survival after UV-C irradiation.

To evaluate this hypothesis, we assessed the production of ROS in response to UV-C irradiation in cotyledons of the double mutant using $\mathrm{H}_{2}$ DCFDA, a reagent that fluoresces in the presence of ROS. As shown in Fig. 5b, lower levels of $\mathrm{H}_{2}$ DCFDA fluorescence were detected in UV-C treated cotyledons ( 30 and $50 \mathrm{~kJ} \mathrm{~m}^{-2}$ ) of the trxf1;trxf2 mutant compared to WT cotyledons, showing that the absence of type- $f$ TRX lead to a reduced ROS production in cotyledons.

Cell viability was also evaluated in roots using propidium iodide (PI) for the detection of dead cells and fluorescein diacetate (FDA) for living cells (Fig. 5c). Roots of single mutants irradiated with $10 \mathrm{~kJ} \mathrm{~m} \mathrm{~m}^{-2} \mathrm{UV}-\mathrm{C}$ had larger FDA-stained sections than WT roots, while roots of the double mutants exhibited slightly larger FDA-stained sections after $30 \mathrm{~kJ} \mathrm{~m}^{-2}$ UV-C irradiation (Fig. 5c-d). This indicated some increase in the number of cells surviving to UV-C in the mutant roots compared to WT roots. However, this effect was very weak in the double mutant, suggesting that loss of type- $f$ TRX expression had a very subtle effect on improving root cell survival in response to UV-C. As shown earlier, germination of seeds (Fig. 1) and seedling development (Supl. Fig. S4 and Table 1) was a bit faster in the double mutant, which may explain the different sensitivity to UV-C.

We also measured chlorophyll level in whole seedlings before and after UV-C irradiation (Fig. 5 e). The relative loss of chlorophyll was $30 \%$ more important in trxf1;trxf2 seedlings compared to WT seedlings, indicating that loss of type- $f$ TRX decreased chlorophyll content in response to UV-C.

UV-C irradiation also induces programmed cell death (PCD) in Arabidopsis seedlings. To evaluate the extent of PCD, we analysed DNA fragmentation, a hallmark of PCD, induced by UV-C in type- $f$ TRX mutants compared to WT (Suppl. data, Fig. S6). DNA fragmentation was slightly increased in all mutants tested when compared to WT, except for trxf2 after $48 \mathrm{~h}$. This may be explained by the fact that this mutant is 
still producing high levels of TRX $f 1$ which can possibly compensate for the loss of TRX $f 2$. This data suggests that loss of type- $f$ TRXs somewhat accelerated PCD induced by UV-C.

Overall, these results indicate that while loss of type- $f$ TRXs could reduce ROS production in cotyledons irradiated by UV-C, it also increased chlorophyll loss and DNA fragmentation induced by UV-C in seedlings. Consequently, the downregulation of type- $f$ TRXs in response to UV-C may only be one of many factors that would contribute to the regulation of cell death and survival in response to UV-C.

\section{Discussion}

\section{Functional redundancy of Arabidopsis type-f thioredoxins}

In contrast to most plant species which contain only one type- $f$ TRX, Arabidopsis thaliana contains two, TRX $f 1$ and TRX f2. The high degree of homology between both isoforms (Collin et al. 2003) and similar expression profile (Suppl. data, Fig. S1) suggest a redundancy of functions, where one isoform could compensate for the other one. We used a genetic approach to study the importance of type- $f$ TRXs in Arabidopsis. The overall phenotype of single $\operatorname{trxf1}$ and trxf2 mutants as well as double trxf1;trxf2 mutant plants were similar to WT plants grown in standard conditions (Thormählen et al. 2013; Yoshida et al. 2015; Naranjo et al. 2016, and this work), suggesting that other plastidic TRXs, such as type-m TRXs could substitute for the absence of type-f TRXs. However, analyses of specific characteristics in single and double mutants, such as photosynthetic electron transport rates, growth in short day conditions, lightregulated carbon metabolism and starch accumulation (Yoshida et al. 2015; Naranjo et al. 2016), supported the functional redundancy hypothesis. In the current work, we showed that the single trxf1-2 mutant and the double trxf1;trxf2 mutant had a faster germination and seedling growth than other single mutants or WT (Table 1; Fig. 2; Suppl. data, Fig. S3). Moreover, starch accumulation was reduced in the single trxf1-4 mutant, but this reduction was significantly more important in the double mutant (Fig. 3; Naranjo et al. 2016), indicating a combined effect of the loss of both type-f TRXs. Finally, loss of both type$f$ TRXs increased sensitivity to oxidative stress induced by MV compared to WT or single mutants. 
While most of these results support the functional redundancy hypothesis, the absence of TRX $f 1$ had a predominant impact during germination compared to the loss of TRX $f 2$. This may be a specific function for TRX $f 1$ or may be due to the higher abundance of TRX $f 1$ compare to that of TRX $f 2$ (Fig. 1; Okegawa and Motohashi 2015).

Implication of type- $f$ TRXs in seed germination and seedling development

The somewhat faster germination and seedling development phenotype observed in the trxf1;trxf2 mutant suggests that Arabidopsis type- $f$ TRXs are involved in these processes. Supporting our results, earlier work had shown in pea and Arabidopsis that expression of type- $f$ TRX gene is high in developing seeds and cotyledons, suggesting that $\operatorname{TRX} f$ may be implicated in seed formation and early germination (Suppl. data, Fig. S1; Fernández-Trijueque et al. 2012; Belin et al. 2015). In particular, histological localization of GUS expression regulated by the pea TRX $f$ promoter in Arabidopsis young etiolated seedlings showed expression at high levels in non-green cotyledons, with increased expression in green cotyledons but very little expression in hypocotyls (Fernández-Trijueque et al. 2012). Similarly, expression of Arabidopsis TRX $f 1$ (and TRXf2 to a lesser extent) was higher in cotyledons than other tissues (Suppl. data Fig. S1; Belin et al. 2015). It was proposed that high expression of TRX $f$ in cotyledons may indicate a role in reserve mobilization during germination (Fernández-Trijueque et al. 2012). However, our results rather suggest that type- $f$ TRXs, and most particularly TRX $f 1$, have a negative impact on seed germination as loss of type- $f$ TRXs stimulated germination and seedling growth (Table 1; Fig. 2; Suppl. data, Fig. S4). This contrasts with the function of specific type- $h$ TRXs (Lozano et al. 1996; Marx et al. 2003; Montrichard et al. 2003; Wong et al. 2004) which stimulate germination by specifically activating reserve mobilization. This also indicates that different types of TRXs may have opposing functions during plant development. Many possibilities can explain how type- $f$ TRXs are implicated in seed germination and early seedling development. One possibility relies on the type- $f$ TRXs regulatory activity on starch accumulation and mobilization. Several enzymes implicated in starch accumulation and degradation are known to be redox regulated, with in vitro or in vivo evidence pointing to $\operatorname{TRX} f$ as the most efficient activator (Sparla et al. 2006; Valerio et al. 2011; Seung et al. 2013). As shown in Fig. 3, the absence of type-f TRXs reduced starch 
accumulation whereas seedling growth was enhanced on sucrose-containing medium (Table 1). It is possible that reduction in starch accumulation in the $\operatorname{tr} f 1$; $\operatorname{tr} x f 2$ mutant increases the availability and/or redirection of some of the sugars in favor of germination and early seedling development before photosynthesis is fully effective.

On another side, there is wide evidence that seed germination is associated with broad change in the redox state of proteins to activate enzymes functional in germination or stimulate reserve mobilization (Rajjou et al. 2012). Hence, loss of TRXs $f$ in mutants most probably has an impact on the cellular redox status, which in turn could perturb seed germination and seedling development. Recent genetic and functional studies on the role of the plastid NADPH-TRX reductase, or NTRC, has suggested the existence of a functional crosstalk between the Fdx/TRX and NTRC systems in chloroplast redox regulation (Thormählen et al. 2015; Nikkanen et al. 2016; Ojeda et al. 2017). In particular, NTRC and other TRXs, such as type- $f$ TRXs, would work in concerted action in processes previously known to be TRX-regulated, including TRX-regulated pathways such as starch synthesis and chlorophyll biosynthesis (Perez-Ruiz et al. 2006; Michalska et al. 2009; Lepistö et al. 2013; Perez-Ruiz et al. 2014; Thormählen et al. 2015; Nikkanen et al. 2016; Ojeda et al. 2017). Moreover, it was shown that the NTRC system involves direct proteinprotein interaction between TRX f1 and NTRC (Thormählen et al. 2015; Nikkanen et al. 2016). Hence, considering the evidence that NTRC and type- $f$ TRXs can act cooperatively in various processes, it is quite possible that loss of type- $f$ TRXs alters NTRC abundance and/or activity, which could in turn perturb the chloroplast redox balance during germination and seedling development.

Seed germination also depends on an adequate production of ROS, while excessive ROS levels are detrimental to seed and seedling viability (Rajjou et al. 2012; El-Maarouf-Bouteau et al. 2013). The loss of type-f TRXs could also have an effect on ROS levels or signaling, which could in turn stimulate seed germination. The hormone ABA is another important factor controlling the equilibrium between seed dormancy and seed germination. In particular, ROS decrease sensitivity to ABA during germination (Liu et al. 2010; El-Maarouf-Bouteau et al. 2013). We have found that the trxf1;trxf2 mutant is more resistant to ABA than WT during early seedling development, which could be due to enhanced ROS production. During 
germination, cells must strictly regulate the production and balance of ROS to respect the so-called "oxidative window" that ensures optimal ROS levels for germination (El-Maarouf-Bouteau et al. 2013). Hence, in parallel to ROS production, cells synthesize redox buffering components and enzymes with antioxidant activities that maintain optimal ROS levels. As described earlier, tomato leaves silenced for TRX $f$ showed oxidative stress symptoms linked with diminished antioxidative enzyme activity (Cheng et al. 2014). It is possible that loss of type- $f$ TRXs decreases antioxidant activity in the seeds, which could lead to a weak increase in ROS production sufficient to stimulate seed germination and early seedling growth slightly above level of WT seeds. Some of the antioxidant activity can be regulated by NTRC which is an electron donor for the catalytic activity of the $\mathrm{H}_{2} \mathrm{O}_{2}$ scavenging thiol-dependent peroxidase 2-cys peroxiredoxin (2-Cys Prx; Moon et al. 2006; Perez-Ruiz et al. 2006; Michalska et al. 2009). As speculated above, a perturbation in NTRC activity caused by loss of type- $f$ TRX could in turn alter the activity of 2-Cys Prx and lead to increased ROS production. Further work will be necessary to address these hypotheses and specifically identify how type- $f$ TRXs regulate seed germination and seedling development.

Involvement of type-f thioredoxins in response to oxidative stress

Animal TRXs are key components of antioxidative systems that protect cells from oxidative stress and cell death (Charruyer et al. 2007; Matsuzawa and Ichijo 2008; Ono et al. 2012). In plants, there is increasing evidence that some TRXs function in protection against oxidative stress. For example, partial silencing of both TRX $f$ and TRX $m$, but not TRX $f$ alone, increased ROS production in pea leaves (Luo et al. 2012). On the other side, overexpression of TRX $m$ increased tolerance to $M V$ in tobacco leaves but overexpression of TRX $f$ plants were as sensitive to MV as WT plants (Rey et al. 2013). Similarly, partial loss of TRX $f$ in tomato leaves enhanced levels of MDA, an indicator of ROS-induced lipid peroxidation, suggesting increased oxidative stress that was associated with decreased activity of antioxidant enzymes (Cheng et al. 2014). Accordingly, we found that seedlings of single and double mutants of trxf1 and trxf2 germinated on the oxidative stress-inducing MV were more sensitive to oxidative stress than WT seedlings, with 30 to $40 \%$ less seedlings with green cotyledons and increased relative chlorophyll loss in the double mutant seedlings when compared to WT (Fig. 4). 
The importance of type- $f$ TRXs in response to stress was also illustrated by results obtained in seedlings exposed to UV-C. In Arabidopsis, UV-C irradiation decreased expression of Arabidopsis type- $f$ TRXs and most notably TRX $f 1$ (Fig. 5a), indicating that type- $f$ TRX abundance was not increased to protect seedlings against oxidative stress induced by UV-C. In fact, we found that loss of type- $f$ TRX decreased ROS production in mutant cotyledons irradiated with UV-C and slightly increased cell survival in UV-C treated roots, suggesting that the absence of type- $f$ TRXs may help protect specific tissues from the redox imbalance that occurs in response to UV-C. However, this was not sufficient to protect seedlings from the overall effects of UV-C treatment as trxf1:trxf2 and trxf1-4 seedlings (and to a lesser extent, trxf2 seedlings) showed slight acceleration of DNA fragmentation in response to UV-C coupled to reduced chlorophyll content in the double mutant (Fig.5; Suppl. data, Fig. S6). Hence, the fact that loss of type-f TRXs stimulated cell death suggests their implication, most probably coordinated with other TRXs, in a fine-tuned regulation of the response to oxidative stress.

\section{Conclusions}

Arabidopsis possesses two type- $f$ TRXs that fulfill some redundant functions, but other chloroplastic TRXs can compensate for the loss of TRX $f$ in processes such as photosynthesis. Results obtained with double type- $f$ TRX mutants suggest that type- $f$ TRXs can be involved in both stimulatory and suppressive activities, depending on the development stage, physiological context and environmental conditions.

At the physiological level, we showed that type-f TRXs, with a predominant contribution from TRX $f 1$, negatively regulate seed germination and early seedling development in a mechanism that has yet been identified. However, loss of type- $f$ TRXs enhanced seedling sensitivity to oxidative stress induced by MV and DNA fragmentation induced by UV-C, indicating that type- $f$ TRXs also contribute in maintaining a cellular environment that provides better tolerance to oxidative stress. Further work will be needed to identify the precise role played by type- $f$ TRXs in young seedlings before photosynthesis becomes fully functional.

\section{Acknowledgements}


We thank Dr. László Szabados for providing the promoter-less luciferase T-DNA insertion collection and Dr. Benoit St-Pierre for the pSCA-eyfg plasmid. This work was supported by the National Sciences and Engineering Council of Canada and the Faculté des Sciences of Université de Sherbrooke to N. Beaudoin.

\section{References}

Alvarado, M. C., Zsigmond, L. M., Kovács, I., Cséplö, A., Koncz, C., and Szabados, L. M. 2004. Gene trapping with firefly luciferase in Arabidopsis. Tagging of stress-responsive genes. Plant Physiol. 134(1): 18-27. doi: $10.1104 /$ pp.103.027151

Aranjuelo, I., Tcherkez, G., Jauregui, I., Gilard, F., Ancín, M., Millán, A. F.-S., Larraya, L., Veramendi, J., and Farran, I. 2015. Alteration by thioredoxin f over-expression of primary carbon metabolism and its response to elevated $\mathrm{CO}_{2}$ in tobacco (Nicotiana tabacum L.). Environ. Exp. Bot. 118: 40-48. doi: $\underline{10.1016 / j . e n v e x p b o t .2015 .05 .008}$

Balsera, M., Uberegui, E., Schürmann, P., and Buchanan, B. B. 2014. Evolutionary development of redox regulation in chloroplasts. Antioxid. Redox Signal. 21(9): 1327-1355. doi: 10.1089/ars.2013.5817

Belin, C., Bashandy, T., Cela, J., Delorme-Hinoux, V., Riondet, C., and Reichheld, J. P. 2015. A comprehensive study of thiol reduction gene expression under stress conditions in Arabidopsis thaliana. Plant Cell Environ. 38(2): 299-314. doi: 10.1111/pce.12276

Bohrer, A. S., Massot, V., Innocenti, G., Reichheld, J. P., Issakidis-Bourguet, E., and Vanacker, H. 2012. New insights into the reduction systems of plastidial thioredoxins point out the unique properties of thioredoxin z from Arabidopsis. J. Exp. Bot. 63(18): 6315-6323. Doi: doi.org/10.1093/jxb/ers283

Buchanan, B. B., Holmgren, A., Jacquot, J.-P., and Scheibe, R. 2012. Fifty years in the thioredoxin field and a bountiful harvest. Biochim. Biophys. Acta, 1820(11): 1822-1829. doi: 10.1016/j.bbagen.2012.07.006

Charruyer, A., Jean, C., Colomba, A., Jaffrézou, J. P., Quillet-Mary, A., Laurent, G., and Bezombes, C. 2007. PKCz protects against UV-C-induced apoptosis by inhibiting acid sphingomyelinase-dependent ceramide production. Biochem. J. 405(1): 77-83. doi: 10.1042/BJ20061528 
Cheng, F., Zhou, Y.-H., Xia, X.-J., Shi, K., Zhou, J., and Yu, J.-Q. 2014. Chloroplastic thioredoxin- $f$ and thioredoxin- $m 1 / 4$ play important roles in brassinosteroids-induced changes in $\mathrm{CO}_{2}$ assimilation and cellular redox homeostasis in tomato. J. Exp. Bot. 65(15): 4335-4347. doi: 10.1093/jxb/eru207 Collin, V., Issakidis-Bourguet, E., Marchand, C., Hirasawa, M., Lancelin, J. M., Knaff, D. B., and MiginiacMaslow, M. 2003. The Arabidopsis plastidial thioredoxins: new functions and new insights into specificity. J. Biol. Chem. 278(26): 23747-23752. doi: 10.1074/jbc.M302077200

Dai, S., Friemann, R., Glauser, D. A., Bourquin, F., Manieri, W., Schürmann, P., and Eklund, H. 2007. Structural snapshots along the reaction pathway of ferredoxin-thioredoxin reductase. Nature, 448(7149): 92-96. doi: $\underline{10.1038 / \text { nature05937 }}$

Danon, A., and Gallois, P. 1998. UV-C radiation induces apoptotic-like changes in Arabidopsis thaliana. FEBS Lett. 437(1-2): 131-136.

Das, P., Nutan, K. K., Singla-Pareek, S. L., and Pareek, A. 2015. Oxidative environment and redox homeostasis in plants: dissecting out significant contribution of major cellular organelles. Front. Environ. Sci. 2: 70. doi: 10.3389/fenvs.2014.00070

Ding, Z., and Friml, J. 2010. Auxin regulates distal stem cell differentiation in Arabidopsis roots. Proc. Natl Acad. Sci. U.S.A. 107(26): 12046-12051. doi: $\underline{10.1073 / p n a s .1000672107}$

El-Maarouf-Bouteau, H., Meimoun, P., Job, C., Job, D., and Bailly, C. 2013. Role of protein and mRNA oxidation in seed dormancy and germination. Front. Plant Sci. 4: 77. doi: 10.3389/fpls.2013.00077

Fernández-Trijueque, J., Barajas-López, J. D., Chueca, A., Cazalis, R., Sahrawy, M., and Serrato, A. J. 2012. Plastid thioredoxins $f$ and $m$ are related to the developing and salinity response of post-germinating seeds of Pisum sativum. Plant Sci. 188-189: 82-88. doi: 10.1016/i.plantsci.2012.01.006

Gazzarrini, S., and McCourt, P. 2001. Genetic interactions between ABA, ethylene and sugar signaling pathways. Curr. Opin. Plant Biol. 4(5): 387-391.

Ikegami, A., Yoshimura, N., Motohashi, K., Takahashi, S., Romano, P. G., Hisabori, T., Takamiya, K.-I., and Masuda, T. 2007. The CHLI1 subunit of Arabidopsis thaliana magnesium chelatase is a target protein of the chloroplast thioredoxin. J. Biol. Chem. 282(27): 19282-19291. doi: 10.1074/jbc.M703324200 
Kim, J.-H., and Lee, C.-H. 2003. Mechanism for photoinactivation of PSII by methyl viologen at two temperatures in the leaves of rice (Oryza sativa L.). J. Plant Biol. 46(1): 10-16.

Lepistö, A., Pakula, E., Toivola, J., Krieger-Liszkay, A., Vignols, F., and Rintamäki, E. 2013. Deletion of chloroplast NADPH-dependent thioredoxin reductase results in inability to regulate starch synthesis and causes stunted growth under short-day photoperiods. J. Exp. Bot. 64(12): 3843-3854. doi: $\underline{10.1093 / \mathrm{jxb} / \mathrm{ert} 216}$

Li, L., Ilarslan, H., James, M. G., Myers, A. M., and Wurtele, E. S. 2007. Genome wide co-expression among the starch debranching enzyme genes AtISA1, AtISA2, and AtISA3 in Arabidopsis thaliana. J. Exp. Bot. 58(12): 3323-3342. doi: $10.1093 / \mathrm{jxb} / \mathrm{erm} 180$

Lichtenthaler, H. K. 1987. Chlorophylls and carotenoids: pigments of photosynthetic biomembranes. Meth. Enzymol. 148: 350-382.

Liu, Y., Ye, N., Liu, R., Chen, M., and Zhang, J. 2010. $\mathrm{H}_{2} \mathrm{O}_{2}$ mediates the regulation of ABA catabolism and GA biosynthesis in Arabidopsis seed dormancy and germination. J. Exp. Bot. 61(11): 2979-2990. doi: $\underline{10.1093 / j x b / e r q 125}$

Lozano, R. M., Wong, J. H., Yee, B. C., Peters, A., Kobrehel, K., and Buchanan, B. B. 1996. New evidence for a role for thioredoxin $h$ in germination and seedling development. Planta, 200(1): 100-106.

Lu, J., and Holmgren, A. 2014. The thioredoxin antioxidant system. Free Radic. Biol. Med. 66: 75-87. doi: $\underline{10.1016 / j . f r e e r a d b i o m e d .2013 .07 .036 ~}$

Luo, T., Fan, T., Liu, Y., Rothbart, M., Yu, J., Zhou, S., Grimm, B., and Luo, M. 2012. Thioredoxin redox regulates ATPase activity of magnesium chelatase $\mathrm{CHLI}$ subunit and modulates redox-mediated signaling in tetrapyrrole biosynthesis and homeostasis of reactive oxygen species in pea plants. Plant Physiol. 159(1): 118-130. doi: 10.1104/pp.112.195446

Marx, C., Wong, J. H., and Buchanan, B. B. 2003. Thioredoxin and germinating barley: targets and protein redox changes. Planta, 216(3): 454-460.

Matsui, M., Oshima, M., Oshima, H., Takaku, K., Maruyama, T., Yodoi, J., and Taketo, M. M. 1996. Early embryonic lethality caused by targeted disruption of the mouse thioredoxin gene. Dev. Biol. 178(1): 179-185. 
Matsuzawa, A., and Ichijo, H. 2008. Redox control of cell fate by MAP kinase: physiological roles of ASK1MAP kinase pathway in stress signaling. Biochim. Biophys. Acta, 1780(11): 1325-1336. doi: 10.1016/j.bbagen.2007.12.011

Meyer, Y., Belin, C., Delorme-Hinoux, V., Reichheld, J. P., and Riondet, C. 2012. Thioredoxin and glutaredoxin systems in plants: molecular mechanisms, crosstalks, and functional significance. Antioxid. Redox Signal. 17(8): 1124-1160. doi: 10.1089/ars.2011.4327

Michalska, J., Zauber, H., Buchanan, B. B., Cejudo, F. J., and Geigenberger, P. 2009. NTRC links built-in thioredoxin to light and sucrose in regulating starch synthesis in chloroplasts and amyloplasts. Proc. Natl. Acad. Sci. U.S.A. 106(24): 9908-9913. doi: 10.1073/pnas.0903559106

Michelet, L., Zaffagnini, M., Morisse, S., Sparla, F., Pérez-Pérez, M. E., Francia, F., Danon, A., Marchand, C. H., Fermani, S., Trost, P., and Lemaire, S. D. 2013. Redox regulation of the Calvin-Benson cycle: something old, something new. Front. Plant Sci. 4: 470. doi: $\underline{10.3389 / f p l s .2013 .00470}$

Mikkelsen, R., Mutenda, K. E., Mant, A., Schürmann, P., and Blennow, A. 2005. $\alpha$-Glucan, water dikinase (GWD): a plastidic enzyme with redox-regulated and coordinated catalytic activity and binding affinity. Proc. Natl. Acad. Sci. U.S.A. 102(5): 1785-1790. doi: 10.1073/pnas.0406674102

Montrichard, F., Alkhalfioui, F., Yano, H., Vensel, W. H., Hurkman, W. J., and Buchanan, B. B. 2009. Thioredoxin targets in plants: the first 30 years. J. Proteomics, 72(3): 452-474. doi: 10.1016/j.jprot.2008.12.002

Montrichard, F., Renard, M., Alkhalfioui, F., Duval, F. D., and Macherel, D. 2003. Identification and differential expression of two thioredoxin $\mathrm{h}$ isoforms in germinating seeds from pea. Plant Physiol. 132(3): 1707-1715. doi: 10.1016/i.jprot.2008.12.002

Moon, J. C., Jang, H. H., Chae, H. B., Lee, J. R., Lee, S. Y., Jung, Y. J., Shin, M. R., Lim, H. S., Chung, W. S., and Yun, D.-J. 2006. The C-type Arabidopsis thioredoxin reductase ANTR-C acts as an electron donor to 2Cys peroxiredoxins in chloroplasts. Biochem. Biophys. Res. Commun. 348(2): 478-484. doi: $\underline{10.1016 / j . b b r c .2006 .07 .088}$ 
Naranjo, B., Diaz-Espejo, A., Lindahl, M., and Cejudo, F. J. 2016. Type-f thioredoxins have a role in the short-term activation of carbon metabolism and their loss affects growth under short-day conditions in Arabidopsis thaliana. J. Exp. Bot. 67(6): 1951-1964. doi: 10.1093/jxb/erw017

Nikkanen, L., Toivola, J., and Rintamäki, E. 2016. Crosstalk between chloroplast thioredoxin systems in regulation of photosynthesis. Plant Cell Environ. 39(8):1691-1705. doi: 10.1111/pce.12718

Nonn, L., Williams, R. R., Erickson, R. P., and Powis, G. 2003. The absence of mitochondrial thioredoxin 2 causes massive apoptosis, exencephaly, and early embryonic lethality in homozygous mice. Mol. Cell. Biol. 23(3): 916-922.

Nordberg, J., and Arnér, E. S. 2001. Reactive oxygen species, antioxidants, and the mammalian thioredoxin system. Free Radic. Biol. Med. 31(11): 1287-1312.

Ojeda, V., Perez-Ruiz, J. M., Gonzalez, M., Najera, V. A., Sahrawy, M., Serrato, A. J., Geigenberger, P., and Cejudo, F. J. 2017. NADPH Thioredoxin Reductase C and Thioredoxins Act Concertedly in Seedling Development. Plant Physiol. 174(3): 1436-1448. doi: 10.1104/pp.17.00481

Okegawa, Y., and Motohashi, K. 2015. Chloroplastic thioredoxin $m$ functions as a major regulator of Calvin cycle enzymes during photosynthesis in vivo. Plant J. 84(5): 900-913. doi: 10.1111/tpj.13049

Ono, R., Masaki, T., Dien, S., Yu, X., Fukunaga, A., Yodoi, J., and Nishigori, C. 2012. Suppressive effect of recombinant human thioredoxin on ultraviolet light-induced inflammation and apoptosis in murine skin. J. Dermatol. 39(10): 843-851. doi: 10.1111/j.1346-8138.2012.01566.x

Perez-Ruiz, J. M., Guinea, M., Puerto-Galan, L., and Cejudo, F. J. 2014. NADPH thioredoxin reductase C is involved in redox regulation of the Mg-chelatase I subunit in Arabidopsis thaliana chloroplasts. Mol. Plant, 7(7): 1252-1255. doi: $10.1093 / \mathrm{mp} / \mathrm{ssu} 032$

Perez-Ruiz, J. M., Spinola, M. C., Kirchsteiger, K., Moreno, J., Sahrawy, M., and Cejudo, F. J. 2006. Rice NTRC is a high-efficiency redox system for chloroplast protection against oxidative damage. Plant Cell, 18(9): 2356-2368. doi: $10.1105 /$ tpc.106.041541

Petrov, V., Hille, J., Mueller-Roeber, B., and Gechev, T. S. 2015. ROS-mediated abiotic stress-induced programmed cell death in plants. Front. Plant Sci. 6: 69. doi: 10.3389/fpls.2015.00069 
Rajjou, L., Duval, M., Gallardo, K., Catusse, J., Bally, J., Job, C., and Job, D. 2012. Seed germination and vigor. Annu. Rev. Plant Biol. 63: 507-533. doi: 10.1146/annurev-arplant-042811-105550

Ray, P. D., Huang, B. W., and Tsuji, Y. 2012. Reactive oxygen species (ROS) homeostasis and redox regulation in cellular signaling. Cell. Signal. 24(5): 981-990. doi: 10.1016/i.cellsig.2012.01.008

Rey, P., Sanz-Barrio, R., Innocenti, G., Ksas, B., Courteille, A., Rumeau, D., Issakidis-Bourguet, E., and Farran, I. 2013. Overexpression of plastidial thioredoxins $f$ and $m$ differentially alters photosynthetic activity and response to oxidative stress in tobacco plants. Front. Plant Sci. 4: 390. doi: $\underline{10.3389 / \text { fpls. } 2013.00390}$

Rouhier, N., Cerveau, D., Couturier, J., Reichheld, J.-P., and Rey, P. 2015. Involvement of thiol-based mechanisms in plant development. Biochim. Biophys. Acta, 1850(8): 1479-1496. doi:

\section{$\underline{10.1016 / j . b b a g e n .2015 .01 .023}$}

Sanz-Barrio, R., Corral-Martinez, P., Ancin, M., Segui-Simarro, J. M., and Farran, I. 2013. Overexpression of plastidial thioredoxin f leads to enhanced starch accumulation in tobacco leaves. Plant Biotechnol. J. 11(5): 618-627. doi: $10.1111 /$ pbi.12052

Schneider, C. A., Rasband, W. S., and Eliceiri, K. W. 2012. NIH Image to ImageJ: 25 years of image analysis. Nature Meth. 9(7): 671. doi: 10.1038/nmeth.2089

Seung, D., Thalmann, M., Sparla, F., Abou Hachem, M., Lee, S. K., Issakidis-Bourguet, E., Svensson, B., Zeeman, S. C., and Santelia, D. 2013. Arabidopsis thaliana AMY3 is a unique redox-regulated chloroplastic $\alpha$-amylase. J. Biol. Chem. 288(47): 33620-33633. doi: 10.1074/jbc.M113.514794

Silver, D. M., Silva, L. P., Issakidis-Bourguet, E., Glaring, M. A., Schriemer, D. C., and Moorhead, G. B. 2013. Insight into the redox regulation of the phosphoglucan phosphatase SEX4 involved in starch degradation. FEBS J. 280(2): 538-548. doi: 10.1111/j.1742-4658.2012.08546.x

Skryhan, K., Cuesta-Seijo, J. A., Nielsen, M. M., Marri, L., Mellor, S. B., Glaring, M. A., Jensen, P. E., Palcic, M. M., and Blennow, A. 2015. The role of cysteine residues in redox regulation and protein stability of Arabidopsis thaliana starch synthase 1. PLoS ONE, 10(9): e0136997. doi:

$\underline{10.1371 / j o u r n a l . p o n e .0136997}$ 
Sparla, F., Costa, A., Lo Schiavo, F., Pupillo, P., and Trost, P. 2006. Redox regulation of a novel plastidtargeted $\beta$-amylase of Arabidopsis. Plant Physiol. 141(3): 840-850. doi: 10.1104/pp.106.079186 Sulpice, R., Pyl, E. T., Ishihara, H., Trenkamp, S., Steinfath, M., Witucka-Wall, H., Gibon, Y., Usadel, B., Poree, F., Piques, M. C., Von Korff, M., Steinhauser, M. C., Keurentjes, J. J., Guenther, M., Hoehne, M., Selbig, J., Fernie, A. R., Altmann, T., and Stitt, M. 2009. Starch as a major integrator in the regulation of plant growth. Proc. Natl. Acad. Sci. U.S.A. 106(25): 10348-10353. doi: 10.1073/pnas.0903478106

Thormählen, I., Meitzel, T., Groysman, J., Öchsner, A. B., von Roepenack-Lahaye, E., Naranjo, B., Cejudo, F. J., and Geigenberger, P. 2015. Thioredoxin $f 1$ and NADPH-dependent thioredoxin reductase $C$ have overlapping functions in regulating photosynthetic metabolism and plant growth in response to varying light conditions. Plant Physiol. 169(3): 1766-1786. doi: 10.1104/pp.15.01122

Thormählen, I., Ruber, J., von Roepenack-Lahaye, E., Ehrlich, S. M., Massot, V., Hümmer, C., Tezycka, J., Issakidis-Bourguet, E., and Geigenberger, P. 2013. Inactivation of thioredoxin $f 1$ leads to decreased light activation of ADP-glucose pyrophosphorylase and altered diurnal starch turnover in leaves of Arabidopsis plants. Plant Cell. Environ. 36(1): 16-29. doi: 10.1111/j.1365-3040.2012.02549.x

Toivola, J., Nikkanen, L., Dahlström, K. M., Salminen, T. A., Lepistö, A., Vignols, F., and Rintamäki, E. 2013. Overexpression of chloroplast NADPH-dependent thioredoxin reductase in Arabidopsis enhances leaf growth and elucidates in vivo function of reductase and thioredoxin domains. Front. Plant Sci. 4: 389. doi: $\underline{10.3389 / \mathrm{fpls} .2013 .00389}$

Valerio, C., Costa, A., Marri, L., Issakidis-Bourguet, E., Pupillo, P., Trost, P., and Sparla, F. 2011. Thioredoxin-regulated $\beta$-amylase (BAM1) triggers diurnal starch degradation in guard cells, and in mesophyll cells under osmotic stress. J. Exp. Bot. 62(2): 545-555. doi: 10.1093/jxb/erq288

Weigel, D., and Glazebrook, J. 2002. Arabidopsis: A Laboratory Manual. Cold Spring Harbor Laboratory Press, New York.

Winter, D., Vinegar, B., Nahal, H., Ammar, R., Wilson, G. V., and Provart, N. J. 2007. An "Electronic Fluorescent Pictograph" browser for exploring and analyzing large-scale biological data sets. PLoS ONE, 2(8): e718. doi: 10.1371/journal.pone.0000718 
Wituszyńska, W., Szechyńska-Hebda, M., Sobczak, M., Rusaczonek, A., Kozłowska-Makulska, A., Witoń, D., and Karpiński, S. 2015. LESION SIMULATING DISEASE 1 and ENHANCE DISEASE SUSCEPTIBILITY 1 differentially regulate UV-C-induced photooxidative stress signalling and programmed cell death in Arabidopsis thaliana. Plant Cell Environ. 38(2): 315-330. doi: 10.1111/pce.12288

Wong, J. H., Cai, N., Tanaka, C. K., Vensel, W. H., Hurkman, W. J., and Buchanan, B. B. 2004. Thioredoxin reduction alters the solubility of proteins of wheat starchy endosperm: an early event in cereal germination. Plant Cell Physiol. 45(4): 407-415.

Yoshida, K., Hara, S., and Hisabori, T. 2015. Thioredoxin selectivity for thiol-based redox regulation of target proteins in chloroplasts. J. Biol. Chem. 290(23): 14278-14288. doi: 10.1074/jbc.M115.647545 


\section{Figure captions}

Fig. 1. Localization of T-DNA insertion in trxf1 and trxf2 mutants.

a) TRX f1 (At3g02730) gene organization and localization of T-DNA insertion for trxf1-2 and trxf1-4. b) TRX $f 2$ (At5g16400) gene organization and localization of T-DNA insertion for trxf2-1. c) Gene expression by RT-PCR of TRX f1, TRXf2 and ACTIN in Wild-type (WT), trxf2-1, trxf1-2 and trxf1-4 mutants. d) Immunoblot analysis using anti-TRX $f 1 / 2$ antibodies. Proteins $(15 \mu \mathrm{g})$ were extracted from leaves of 4 wk-old plants grown in long days. Relative amount (\%) of type- $f$ TRX is presented in comparison to WT levels set at $100 \%$ TRX $f$ levels were quantified by normalizing the intensity of the $\operatorname{TRX} f$ band with the intensity of the Coomassie stained gel.

Fig. 2. Seed germination and seedling development in trxf1 and trxf2 mutants on GM and in response to ABA.

a) Percentage of seed germination in trxf1 and trxf2 single and double mutant genotypes compared to WT. b) Percentage of seed germination (left - day 2) and green cotyledons (right - day 5) on ABA in trxf1 and trxf2 single and double mutant genotypes compared to WT. Percentage of germination was calculated based on the total number of seeds tested. Percentage of seed germination after 10 days is presented in Suppl. data, Table S2. At least 100 seeds were analysed at the same time for each genotype. Values are representative of three independent experiments. Significant differences with WT are indicated by $*=p<0.05$, Fisher's exact test.

Fig. 3. Decreased starch content in the trxf1;trxf2 double mutant.

a) Detection of starch using Lugol's staining in 10-day-old rosette, bar $=1 \mathrm{~cm}$. b) Microscopy analysis of starch deposition in Lugol-stained root apices, bar $=20 \mu \mathrm{m}$. c) Microscopy analysis of starch deposition in Lugol-stained upper-root hypocotyl sections, bar $=200 \mu \mathrm{m}$. d) Relative amount of starch in leaves of 3week-old seedlings in the indicated genotypes compared to WT. Values represent the mean \pm SEM of 
three independent experiments each including 15 plants. Significant differences with WT are indicated by $*=p<0.01, t$-test.

Fig. 4. Increased sensitivity to methyl viologen (MV) in trxf1 and trxf2 mutants.

a) Percentage of green cotyledons in 8-day-old seedlings of trxf1 and trxf2 single and double mutant genotypes grown on GM supplemented with $0,0.5$ or $1.0 \mu \mathrm{M} \mathrm{MV}$. Values represent the mean of three experiments with at least 100 seedlings each \pm SEM. Significant differences with WT are indicated by $*=p$ $<0.05, t$-test. b) Relative chlorophyll content in seedlings grown on $0.5 \mu \mathrm{M} \mathrm{MV}$ for 10 days compared to seedlings grown on control medium. Values are the mean of three experiments with at least 15 seedlings each \pm SEM. Significant differences with WT are indicated by $*=p<0.05, t$-test.

Fig. 5. Effects of UV-C irradiation on trxf1 and trxf2 single and double mutants.

a) Upper panel: Gene expression of $T R X f 1, T R X f 2$ and $A C T I N$ analysed by RT-PCR in non-treated (NT) seedlings, just after treatment $(0 \mathrm{~h})$ and 24 or $48 \mathrm{~h}$ after UV-C exposure $\left(30 \mathrm{~kJ} \mathrm{~m}^{-2}\right)$ in the indicated genotypes; Lower panel: Immunoblot analysis using anti-TRX $f 1 / 2$ antibodies. Soluble proteins (10 $\mu \mathrm{g})$ were extracted from WT seedlings $24 \mathrm{~h}$ after UV-C irradiation (UV-C) or untreated seedlings (control). Relative amount (\%) of type- $f$ TRX is indicated in comparison to WT levels set à $100 \%$. TRX $f$ levels were quantified by normalizing the intensity of the TRX $f$ band with the intensity of the Coomassie stained gel. b) Epifluorescence microscopy analyses of cotyledons $24 \mathrm{~h}$ after UV-C treatment (10, 30 and $\left.50 \mathrm{~kJ} \mathrm{~m}^{-2}\right)$. ROS production is detected by $\mathrm{H}_{2}$ DCFDA fluorescence (yellow to light green - left and right panels). Bar = $200 \mu \mathrm{m}$. c) Epifluorescence microscopy analyses of roots $24 \mathrm{~h}$ after UV-C treatment (10 and $30 \mathrm{~kJ} \mathrm{~m} \mathrm{~m}^{-2}$. Living cells are green (FDA fluorescence) and dead cells are red (PI fluorescence). Bar $=200 \mu \mathrm{m}$. d) Percentage of root surface with living cells as detected by FDA fluorescence (lower bar, green) compared to dead cells as detected by PI fluorescence (upper bar, red) from analyses presented in c. e) Relative chlorophyll content of seedlings irradiated with 5 or $15 \mathrm{~kJ} \mathrm{~m}^{-2} \mathrm{UV}-\mathrm{C}$ compared to untreated seedlings. Values are the mean of three experiments with at least 30 seedlings each \pm SEM. Significant differences with WT are indicated by $*=p<0.05, t$-test. 
Table 1. Average weight of a single 10-day-old seedling.

\begin{tabular}{lcccc}
\hline Genotype & WT Col-0 & trxf2-1 & trxf1-4 & trxf1;trxf2 \\
\hline Weight (mg) & $7.1 \pm 1.0^{\mathrm{a}}$ & $7.5 \pm 0.4^{\mathrm{ab}}$ & $7.8 \pm 1.2^{\mathrm{ab}}$ & $9.2 \pm 0.9^{\mathrm{b}}$ \\
\hline
\end{tabular}

Each value represents the average weight \pm SD of a single seedling calculated from three distinct pools of at least 15 10-day-old seedlings. Different letters between samples within a single line indicate significantly different values $(p<0.05)$ using one-way ANOVA followed by Holm-Sidak's test for multiple comparison. 

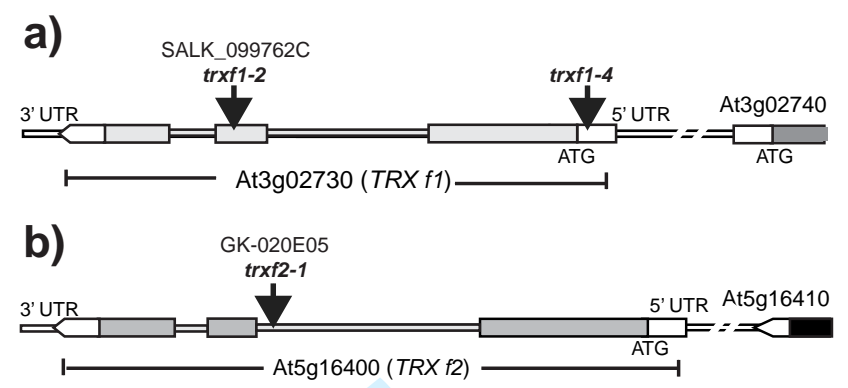

c)

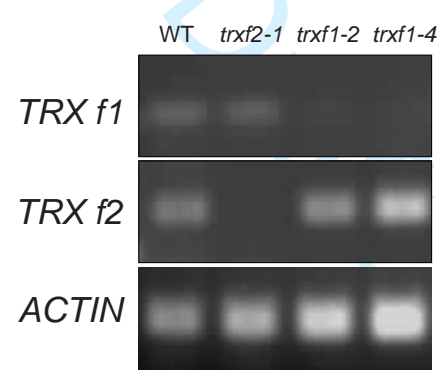

d)

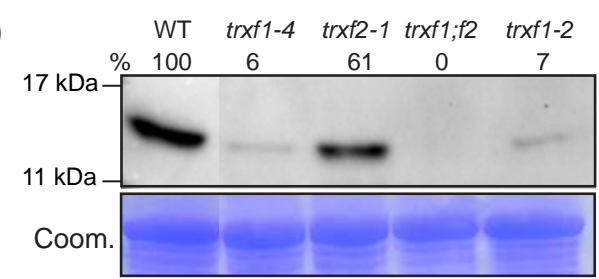

https://mc06.manuscriptcentral.com/botany-pubs 
a)

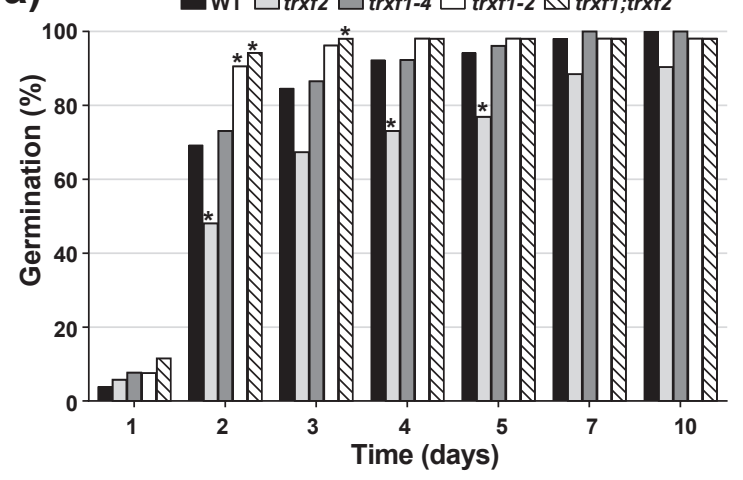

b)

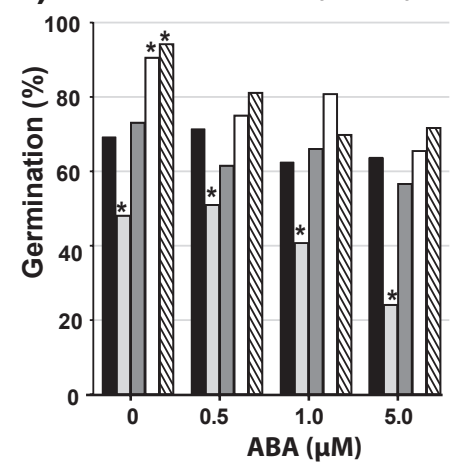

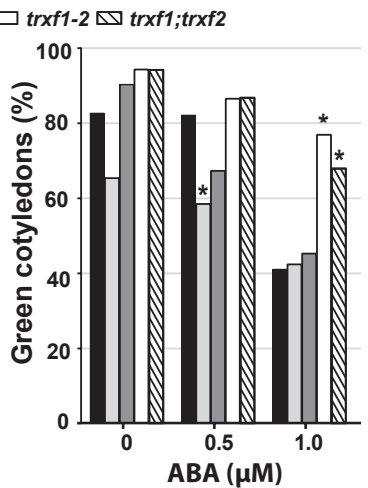


a)

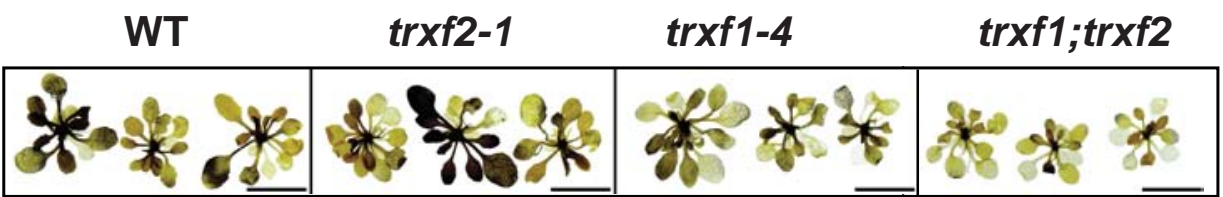

b)

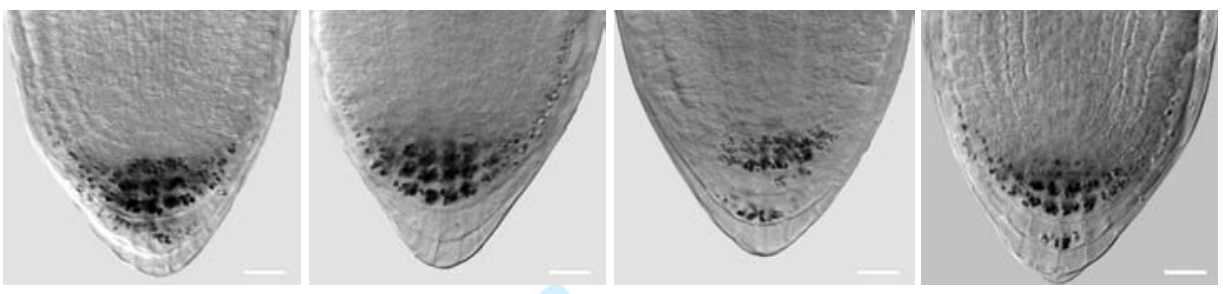

c)

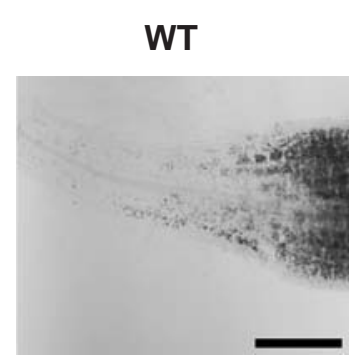

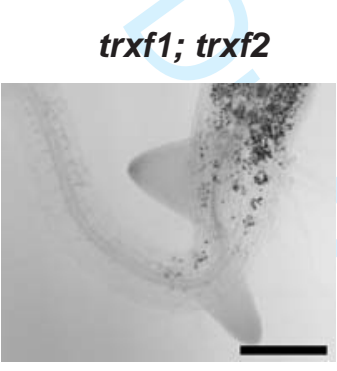

d)

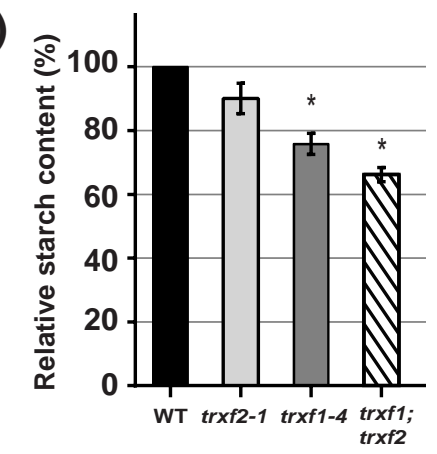


a)

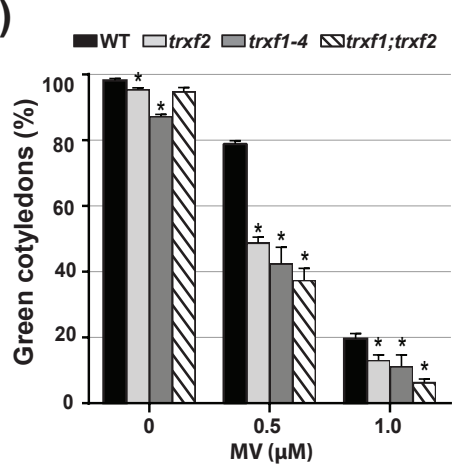

b)

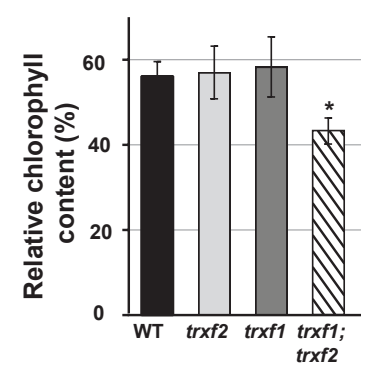




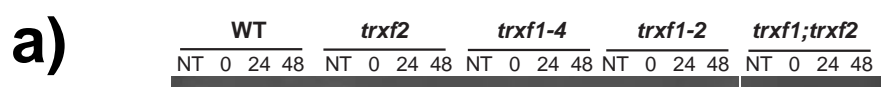
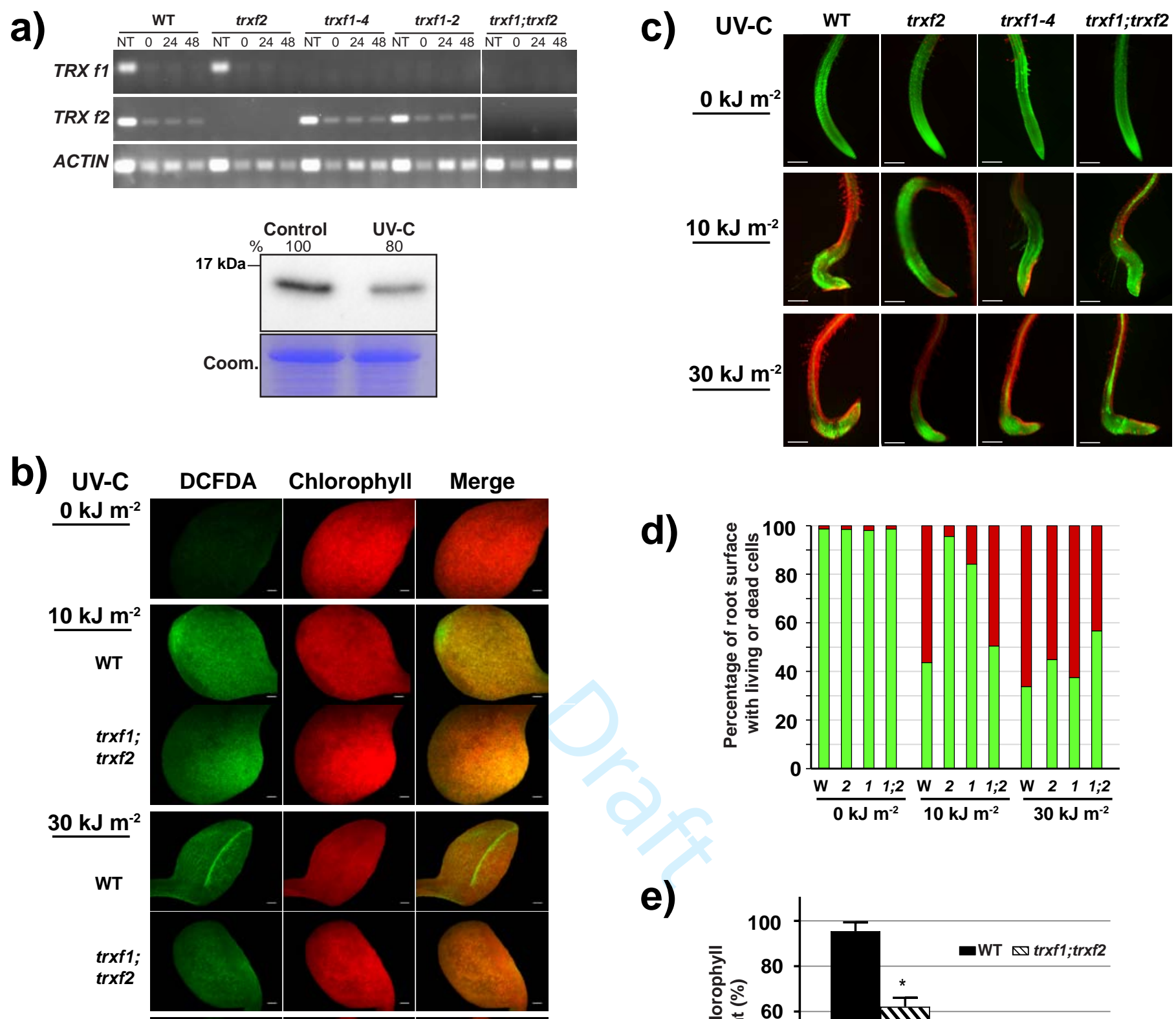

d)
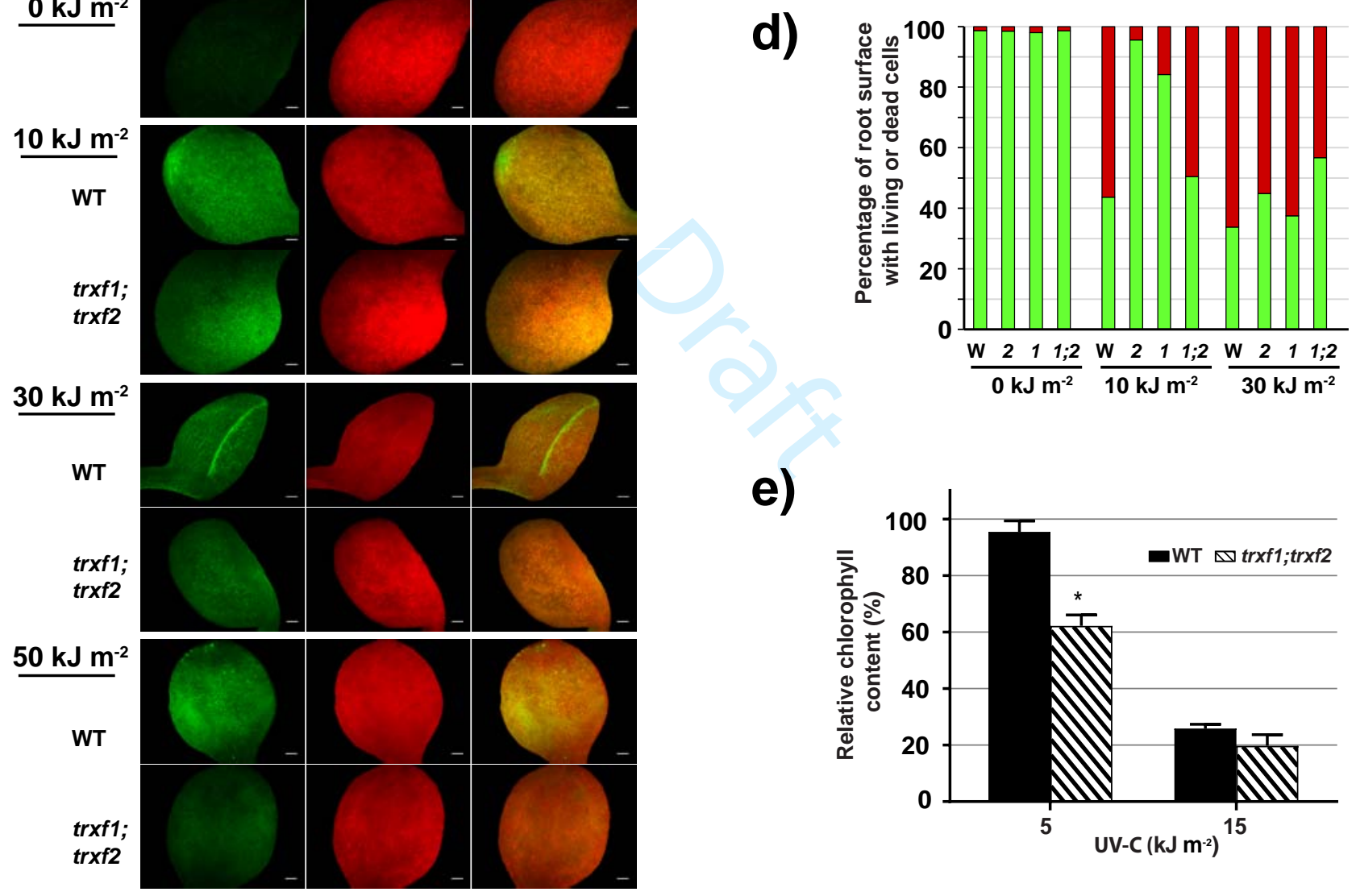

e)

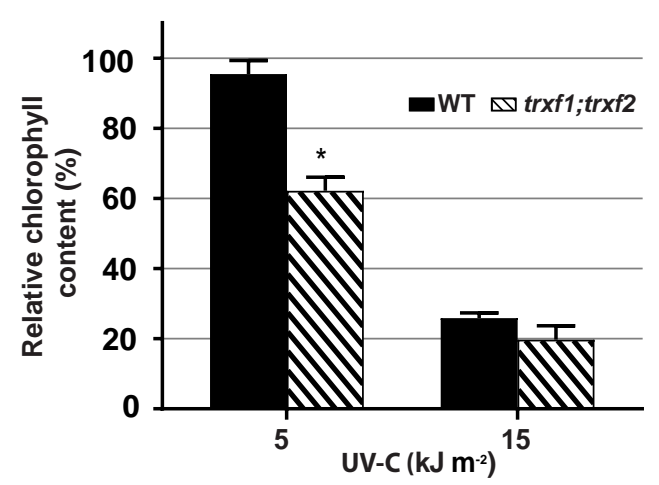

